\title{
Aplicación de la clasificación de Robson para el análisis de la tasa de cesárea en el Hospital Doctor Peset
}

\section{Review of cesarean rate in Hospital Doctor Peset using the Robson classification}

\author{
Guillermo S. Martínez ${ }^{1 *}$, Gema Zomeño ${ }^{1}$, María P. Tarrazó1, Amparo Garayoa ${ }^{1}$, Carolina Sanchez ${ }^{2}$ \\ y María Reyes-Balanzá ${ }^{1}$ \\ ${ }^{1}$ Servicio de Ginecología y Obstetricia; ${ }^{2}$ Servicio de Matronas Paritorio. Hospital Doctor Peset, Valencia, España
}

\section{Resumen}

Objetivo: Analizar las cesáreas realizadas en nuestro centro agrupándolas según la clasificación de Robson para establecer medidas que permitan reducir la tasa de cesáreas. Método: Auditoría prospectiva de los nacimientos asistidos en el Hospital Doctor Peset en el año 2019 mediante la clasificación de Robson. Resultados: Se han analizado 1113 nacimientos con una tasa de cesárea del 25.3\%. El grupo que más contribuyó al total de cesáreas realizadas, con un $34.4 \%$, fue el $2 A$ (nulíparas con feto único en presentación cefálica, de 37 semanas o más de embarazo, que iniciaron el parto mediante inducción). En segundo lugar, el grupo 5 (multíparas con al menos una cesárea previa, con un feto único en presentación cefálica, de 37 semanas o más de embarazo), con un 20.1\%. Las inducciones en nulíparas multiplican por tres la tasa de cesárea respecto a las nulíparas que inician el trabajo de parto de manera espontánea. Conclusiones: La clasificación de Robson es una herramienta que permite clasificar y analizar de manera sencilla los grupos en los que implantar medidas para reducir el número de cesáreas realizadas. Analizar las indicaciones de inducción y revisar los protocolos de actuación podría suponer una disminución sustancial en la tasa de cesáreas en nuestro centro.

Palabras clave: Tasa de cesáreas. Clasificación Robson. Auditoría. Contribución.

\section{Abstract}

Objective: To analyze the caesarean deliveries attended in our hospital, grouping them according to the Robson Classification System and to establish measures in order to reduce caesarean delivery rates. Method: Prospective study of all the deliveries attended at Hospital Doctor Peset in 2019 using the Robson classification. Results: A total of 1113 births have been analyzed with a total cesarean section rate of $25.3 \%$. The largest contribution to the total cesarean delivery rate with $34.4 \%$ was group $2 A$ (nulliparous women with a single fetus in cephalic presentation, 37 weeks or more pregnant who started labor by induction). Secondly, group 5 (multiparous women with at least previous cesarean section, with single cephalic fetus, 37 weeks or more pregnant) which represents the $20.1 \%$ of the total. Inductions in nulliparas multiply the cesarean section rate by 3 compared to nulliparas that initiate labor spontaneously. Conclusions: Robson's classification is a tool that allows to easily classify and analyze the groups in which to implement measures to reduce the number of caesarean sections performed. Analyzing the induction indications and reviewing action protocols could suppose a substantial decrease in the caesarean section rate in our center.

Key words: Cesarean section rate. Robson classification. Audit. Contribution.

Correspondencia:

*Guillermo S. Martínez

E-mail: guillermomarfer@gmail.com
Disponible en internet: $27-10-2021$ Rev Chil Obstet Ginecol. 2021;86(4):353-359

www. rechog.com 0048-766X / @ 2021 Sociedad Chilena de Obstetricia y Ginecología. Publicado por Permanyer. Éste es un artículo open access bajo la licencia CC BY-NC-ND (https://creativecommons.org/licenses/by-nc-nd/4.0/). 


\section{Introducción}

La Organización Mundial de la Salud (OMS) considera la tasa de cesáreas como un indicador de calidad asistencial en la atención materno-fetal. Este organismo recomendó en 1985 una tasa de cesáreas del 10-15\% . Sin embargo, en las últimas décadas se ha producido un aumento sustancial de dicha tasa tanto en España como en el resto de los países desarrolla$\operatorname{dos}^{2-4}$. Según datos del Ministerio de Sanidad, la tasa de cesáreas en España en 1996 era del 19.2\% y en 2011 alcanzó el $24.9 \%$. Por su parte, en la Comunidad Valenciana, en 2017 era del $30.24 \%{ }^{5}$. Estos datos distan del objetivo marcado por la OMS, por lo que resulta fundamental implantar medidas para reducir el número de cesáreas realizadas.

En 2001, M. Robson propuso un sistema de clasificación según las características obstétricas que permite la monitorización y la auditoría de la tasa de cesáreas $^{6}$. La OMS, en una revisión sistemática realizada en 2011 y confirmada en 2014, establece esta clasificación como la herramienta más apropiada para monitorizar, auditar y comparar las tasas de cesáreas $^{7-9}$. En 2016, la Federación Internacional de Ginecología y Obstetricia (FIGO) ${ }^{10}$, siguiendo la misma línea, reconoció la clasificación de Robson como el sistema más útil para el análisis de las cesáreas.

La clasificación de Robson ordena a las pacientes en 10 grupos de acuerdo con unas características obstétricas bien definidas y recogidas de manera sistemática, y permite un análisis conceptualmente fácil, reproducible y que posibilita realizar estudios de manera tanto prospectiva como retrospectiva ${ }^{8}$. Los cuatro factores que se tienen en cuenta para la clasificación son la categoría del embarazo (simple/múltiple y la presentación), la edad gestacional, los antecedentes obstétricos y el inicio del trabajo de parto (espontáneo, inducido o cesárea previa trabajo de parto). Se trata de una clasificación robusta, puesto que los grupos son excluyentes e incluyentes al mismo tiempo, de manera que cada mujer puede ser clasificada en un solo grupo (Tabla 1). Este último aspecto permite salvar un problema que aparece de manera frecuente cuando se clasifican las cesáreas en función de su indicación.

El objetivo de este estudio es auditar nuestro centro aplicando la clasificación en 10 grupos de Robson, con el fin de establecer medidas que permitan reducir la tasa de cesáreas en nuestro hospital.
Tabla 1. Clasificación por grupos de Robson

\begin{tabular}{|c|c|}
\hline Grupos & Pacientes incluidas \\
\hline 1 & $\begin{array}{l}\text { Nulíparas con gestación única de } 37 \text { semanas o } \\
\text { más, en presentación cefálica, que inician trabajo } \\
\text { espontáneo de parto }\end{array}$ \\
\hline $2 A$ & $\begin{array}{l}\text { Nulíparas con gestación única de } 37 \text { semanas o } \\
\text { más, en presentación cefálica, en las que se realiza } \\
\text { inducción }\end{array}$ \\
\hline $2 B$ & $\begin{array}{l}\text { Nulíparas con gestación única de } 37 \text { semanas o } \\
\text { más, en presentación cefálica, en las que se realiza } \\
\text { cesárea previa al inicio de trabajo de parto }\end{array}$ \\
\hline 3 & $\begin{array}{l}\text { Multíparas con gestación única de } 37 \text { semanas o } \\
\text { más, en presentación cefálica, que inician trabajo } \\
\text { de parto espontáneo }\end{array}$ \\
\hline $4 \mathrm{~A}$ & $\begin{array}{l}\text { Multíparas con gestación única de } 37 \text { semanas o } \\
\text { más, en presentación cefálica, en las que se realiza } \\
\text { inducción }\end{array}$ \\
\hline $4 B$ & $\begin{array}{l}\text { Multíparas con gestación única de } 37 \text { semanas o } \\
\text { más, en presentación cefálica, en las que se realiza } \\
\text { cesárea previa al inicio de trabajo de parto }\end{array}$ \\
\hline 5 & $\begin{array}{l}\text { Pacientes con cesárea previa y gestación única de } \\
37 \text { semanas o más, en presentación cefálica }\end{array}$ \\
\hline 6 & Nulíparas con feto único en presentación podálica \\
\hline 7 & $\begin{array}{l}\text { Multíparas (incluyendo cesárea previa) con feto } \\
\text { único en presentación podálica }\end{array}$ \\
\hline 8 & $\begin{array}{l}\text { Todas las pacientes con embarazo múltiple, } \\
\text { incluidas aquellas con cesárea previa }\end{array}$ \\
\hline 9 & $\begin{array}{l}\text { Todas las pacientes con feto único en posición } \\
\text { trasversa u oblicua, incluidas aquellas con cesárea } \\
\text { previa }\end{array}$ \\
\hline 10 & $\begin{array}{l}\text { Todas las pacientes con feto único en presentación } \\
\text { cefálica de menos de } 37 \text { semanas de gestación, } \\
\text { incluidas aquellas con cesárea previa }\end{array}$ \\
\hline
\end{tabular}

\section{Método}

El trabajo se realizó en el Hospital Doctor Peset, de Valencia (España), dependiente de la Conselleria de Sanitat y Salut Pública de la Comunitat Valenciana, en el que solo se atienden partos a partir de la semana 32 de gestación por no contar con unidad de cuidados intensivos neonatal. Es un estudio observacional, descriptivo y prospectivo, en el que se clasifican de acuerdo con la clasificación en 10 grupos de Robson los nacimientos en dicho centro en 2019. Se incluyen en el estudio todos aquellos nacimientos con recién nacido vivo a partir de la semana 32 de gestación. Se excluyen los óbitos fetales.

Los datos recogidos para el análisis son: gestación única o múltiple, edad gestacional, presentación fetal, 
paridad, cesárea previa, inicio de trabajo de parto y forma de finalización.

La información se analizó mediante una hoja de cálculo de Excel y el programa estadístico IBM SPSS Statistics 20.0 .

\section{Resultados}

Durante el año 2019, en el Hospital Doctor Peset se atendieron 1113 nacimientos. De ellos, en 282 (25.3\%) se realizó cesárea y 831 (74.7\%) fueron por vía vaginal. Por tanto, la tasa de cesáreas del año 2019 en nuestro centro fue del $25.3 \%$. Del total de nacimientos, $1081(97.1 \%)$ fueron a término y solo $32(2.9 \%)$ fueron pretérmino. Además, 1089 (97.8\%) de los partos fueron de gestación única y 24 (2.2\%) de gestación múltiple (Tabla 2).

La tabla 3 recoge la distribución de los grupos clínicos de Robson, la tasa de cesáreas de cada uno de ellos y su aportación al total de nacimientos y de cesáreas. Podemos observar que los grupos más numerosos son el grupo 3 (multíparas sin cesárea previa, con un feto en presentación cefálica, de 37 o más semanas de embarazo, que han iniciado el parto de forma espontánea) con un $25.3 \%$ de los nacimientos, el grupo $2 \mathrm{~A}$ (primíparas con feto en presentación cefálica, de 37 semanas o más de embarazo, que han iniciado el parto mediante inducción) con un $24.1 \%$ de los nacimientos, y el grupo 1 (primíparas con feto en presentación cefálica, de 37 semanas o más de embarazo, que han iniciado el parto de manera espontánea) con un $22.2 \%$. En total, entre los tres grupos representan el $71.6 \%$ de los nacimientos de nuestro centro. En contraposición, los grupos menos numerosos son el grupo 9 (todas las mujeres con feto único en posición transversa u oblicua) y el grupo 4B (multíparas sin cesárea previa, gestación única, presentación cefálica, más de 37 semanas gestación, cesárea previa trabajo de parto), que representan un $0.2 \%$ y un $0.9 \%$, respectivamente, del total de los nacimientos.

En lo que respecta a la tasa de cesáreas, existen grupos que por definición presentan una tasa del $100 \%$, como son aquellos en los que se realiza la cesárea previa al trabajo de parto: el 2B (nulíparas, gestación única, cefálica, > 37 semanas gestación, cesárea previa a trabajo de parto) y el 4B (multíparas, gestación única, cefálica, > 37 semanas gestación, cesárea previa a trabajo de parto). Del mismo modo, las gestaciones con presentación oblicua o transversa, que pertenecen al grupo 9, también tienen una tasa de cesáreas del $100 \%$. Sin embargo, estos tres grupos
Tabla 2. Resumen de nacimientos en 2019

$\begin{array}{lc}\text { Total nacimientos } & 1113 \\ \text { Parto vaginal } & 831(74.5 \%) \\ \text { Cesárea } & 282(25.3 \%) \\ \text { Gestación simple } & 1089(97.8 \%) \\ \text { Embarazo a término } & 1081(97.1 \%)\end{array}$

representan un porcentaje bajo del total de las cesáreas.

Analizando los casos del grupo 7 (multíparas con fetos en presentación podálica, incluyendo cesárea anterior), este también presenta una tasa de cesáreas del 100\%. Del mismo modo, el grupo 6 (primíparas con fetos en presentación podálica) también obtuvo un valor cercano al $100 \%$ (96.2\%), puesto que hubo un nacimiento en presentación podálica que se atendió por vía vaginal al acudir a urgencias en periodo activo de parto avanzado y estar de guardia en ese momento personal entrenado para ello.

En cuanto a los grupos más numerosos, observamos tasas de cesáreas elevadas en el grupo 5 (multíparas con al menos una cesárea previa, con feto único en presentación cefálica, de 37 semanas o más de embarazo), del $53.8 \%$, y en el grupo $2 \mathrm{~A}$ (primíparas con feto en presentación cefálica, de 37 semanas 0 más de embarazo, que han iniciado el parto mediante inducción), del $36.2 \%$.

En la contribución al total de las cesáreas realizadas, observamos que el grupo que más aporta es el 2A, que supone el $34.4 \%$ de las cesáreas practicadas; en segundo lugar, el grupo 5, con un $20.2 \%$ de las cesáreas; y en tercer lugar, el grupo 1 con una contribución a las cesáreas del $10.6 \%$. En total, estos grupos representan el $65.2 \%$ de las cesáreas realizadas.

\section{Discusión}

El incremento persistente de la tasa de cesáreas obliga a la implementación de herramientas que permitan analizar las circunstancias y los grupos de mujeres en que se produce un mayor número de cesáreas, con la finalidad de poder intervenir en ellos. La aplicación de la clasificación en diez grupos de Robson ${ }^{6}$ nos ha permitido identificar los grupos de mujeres que más aportan al total de nacimientos, que presentan una mayor tasa de cesáreas y que más contribuyen al total de las cesáreas realizadas en nuestro centro.

El análisis de los datos muestra una tasa de cesáreas del $25.3 \%$, porcentaje que va en consonancia con la elevada tasa de cesáreas existente en la actualidad 
Rev Chil Obstet Ginecol. 2021;86(4)

Tabla 3. Porcentajes de cesáreas por grupos de la clasificación de Robson

\begin{tabular}{|c|c|c|c|c|}
\hline $\begin{array}{l}\text { Grupo de } \\
\text { Robson }\end{array}$ & $\begin{array}{c}\text { Nacimientos } \\
\text { (partos/cesáreas) }\end{array}$ & Tamaño del grupo (\%) & Tasa de cesáreas (\%) & $\begin{array}{c}\text { Contribución al total de } \\
\text { cesáreas (\%) }\end{array}$ \\
\hline 1 & $247(217 / 30)$ & $22.2 \%$ & $12.2 \%$ & $10.6 \%$ \\
\hline $2 A$ & $268(171 / 97)$ & $24.1 \%$ & $36.2 \%$ & $34.4 \%$ \\
\hline $2 B$ & $7(0 / 7)$ & $0.6 \%$ & $100 \%$ & $2.5 \%$ \\
\hline 3 & $282(272 / 10)$ & $25.3 \%$ & $3.6 \%$ & $3.6 \%$ \\
\hline $4 \mathrm{~A}$ & $97(83 / 14)$ & $8.7 \%$ & $14.4 \%$ & $5 \%$ \\
\hline $4 B$ & $10(0 / 10)$ & $0.9 \%$ & $100 \%$ & $3.6 \%$ \\
\hline 5 & $106(49 / 57)$ & $9.5 \%$ & $53.8 \%$ & $20.2 \%$ \\
\hline 6 & $26(1 / 25)$ & $2.3 \%$ & $96.2 \%$ & $8.9 \%$ \\
\hline 7 & $12(0 / 12$ & $1.1 \%$ & $100 \%$ & $4.3 \%$ \\
\hline 8 & $24(14 / 10)$ & $2.2 \%$ & $41.7 \%$ & $3.5 \%$ \\
\hline 9 & $2(0 / 2)$ & $0.2 \%$ & $100 \%$ & $0.7 \%$ \\
\hline 10 & $32(24 / 8)$ & $2.9 \%$ & $25 \%$ & $2.8 \%$ \\
\hline Total & $1113(831 / 282)$ & $100 \%$ & - & $100 \%$ \\
\hline
\end{tabular}

Tabla 4. Comparación del presente estudio con otros estudios realizados

\begin{tabular}{|c|c|c|c|c|}
\hline $\begin{array}{l}\text { Grupo de } \\
\text { Robson }\end{array}$ & $\begin{array}{l}\text { Hospital Doctor Peset } \\
\text { Tamaño del grupo /tasa de } \\
\text { cesáreas }\end{array}$ & $\begin{array}{l}\text { Hospital La Inmaculada } \\
\text { Tamaño del grupo/tasa de } \\
\text { cesáreas }\end{array}$ & $\begin{array}{l}\text { Hospital de Manises } \\
\text { Tamaño del grupo/tasa de } \\
\text { cesáreas }\end{array}$ & $\begin{array}{l}\text { Hospital de Manacor } \\
\text { Tamaño del grupo/tasa de } \\
\text { cesáreas }\end{array}$ \\
\hline 1 & $22.2 \% / 12.2 \%$ & $31.2 \% / 9 \%$ & $26.8 \% / 5.9 \%$ & $32 \% / 9.8 \%$ \\
\hline $2 A$ & $24.1 \% / 36.2 \%$ & \multirow[b]{2}{*}{$13.6 \% / 30 \%$} & \multirow[b]{2}{*}{$18.6 \% / 24.1 \%$} & \multirow[b]{2}{*}{$12.1 \% / 36 \%$} \\
\hline $2 B$ & $0.6 \% / 100 \%$ & & & \\
\hline 3 & $25.3 \% / 3.6 \%$ & $32.2 \% / 2.19 \%$ & $28.3 \% / 2.8 \%$ & $33.6 \% / 4.6 \%$ \\
\hline $4 \mathrm{~A}$ & $8.7 \% / 14.4 \%$ & \multirow{2}{*}{$7.2 / 10.7 \%$} & \multirow{2}{*}{$9.3 \% / 12.5 \%$} & \multirow{2}{*}{$7.9 \% / 23.7 \%$} \\
\hline $4 \mathrm{~B}$ & $0.9 \% / 100 \%$ & & & \\
\hline 5 & $9.5 \% / 53.8 \%$ & $7.4 \% / 42.2 \%$ & $9.9 \% / 44.1 \%$ & $3.8 \% / 44.6 \%$ \\
\hline 6 & $2.3 \% / 96.2 \%$ & $1.6 \% / 94.7 \%$ & $1.5 \% / 97.9 \%$ & $2 \% / 100 \%$ \\
\hline 7 & $1.1 \% / 100 \%$ & $1.3 \% / 98.2 \% \% /$ & $0.4 \% / 83.3 \%$ & $1.8 \% / 100 \%$ \\
\hline 8 & $2.2 \% / 41.7 \%$ & $1.5 \% / 67.4 \%$ & $1.6 \% / 63.5 \%$ & $1 \% / 56 \%$ \\
\hline 9 & $0.2 \% / 100 \%$ & $0.1 \% / 100 \%$ & $0 / 100 \%$ & $0.4 \% / 100 \%$ \\
\hline 10 & $2.9 \% / 25 \%$ & $3.9 \% / 20.2 \%$ & $3.6 \% / 24.6 \%$ & $5.4 \% / 22.8 \%$ \\
\hline
\end{tabular}

y con la publicada por el Ministerio de Sanidad en $2011(24.9 \%)^{5}$. Estos valores están alejados de las recomendaciones de la OMS de 1985. Asimismo, resulta llamativo que la tasa de cesáreas en nuestro hospital es más alta que la publicada por otros centros españoles ${ }^{11-13}$ en los que aplicaron la clasificación de
Robson y cuyas tasas de cesáreas se ajustan más a las recomendaciones actuales.

El tamaño de los grupos es similar al descrito en otros estudios nacionales ${ }^{11-13}$, siendo el que más aporta al total de nacimientos el grupo 3 (Tabla 4). Sin embargo, encontramos aspectos diferenciales que 
pueden contribuir a una mayor tasa de cesáreas. Así, en nuestro centro vemos que el grupo 2A (primíparas con feto en cefálica, de 37 semanas o más de embarazo, que han iniciado el parto mediante inducción) es más numeroso que el grupo 1 , mientras que en el resto de las series es a la inversa (Tabla 4). A esta circunstancia se añade que en nuestro estudio la tasa de cesáreas en el grupo 2A triplica la del grupo 1. De hecho, analizando el grupo $2 \mathrm{~A}$ es llamativo que supone el $24.1 \%$ de los nacimientos, representa un tercio de las cesáreas realizadas $(34.4 \%)$ y se practica cesárea en uno de cada tres casos, lo que supone una tasa de cesáreas del $36.2 \%$. Con todo ello, resulta imperativo por parte de nuestro servicio la implantación de medidas para disminuir el número de inducciones en primíparas y su tasa de cesáreas. La revisión en sesión de las indicaciones de inducción o la actualización del protocolo de inducción al parto pueden llevarnos a una reducción de la tasa de cesáreas. En este sentido, el hecho de que los grupos 1 y 3 (primíparas/multíparas con feto en cefálica, de 37 semanas o más de embarazo, que han iniciado el parto de manera espontánea) presenten tasas de cesáreas del $12.2 \%$ y el $3.6 \%$ es esperanzador para reducir la tasa de cesáreas con las citadas medidas.

El grupo 5 (multíparas con al menos una cesárea previa, con feto único en presentación cefálica, de 37 semanas o más de embarazo) tiene unas características heterogéneas y es el segundo que más contribuye al total de las cesáreas realizadas, con un $20.2 \%$, a pesar de que solo supone un $9.5 \%$ del total de los nacimientos en nuestro hospital (Tabla 3). Asimismo, su tasa de cesáreas es del $53.8 \%$, porcentaje ligeramente superior al presentado en los estudios de ámbito nacional ${ }^{11-13}$, pero inferior a la tasa de cesáreas descrita por Robson ${ }^{6}$ y en otras publicaciones internacionales ${ }^{14-16}$. Con todo ello, es un grupo sobre el que también es necesario realizar un análisis pormenorizado y aplicar medidas que permitan reducir tanto su tasa de cesáreas como el número de pacientes que lo integran, pues resulta importante resaltar que el incremento de mujeres sometidas a una primera cesárea va a aumentar el número de pacientes que integran el grupo 5.

De esta manera, si se realiza un análisis más exhaustivo de las pacientes que forman parte del grupo 5 (multíparas con al menos una cesárea previa, con feto único en presentación cefálica, de 37 semanas o más de embarazo) podemos dividirlo en si presentan una o dos cesáreas previas, así como en si han tenido o no, además de la cesárea previa, un parto por vía vaginal
Tabla 5. Análisis del grupo 5 de Robson (multíparas con al menos una cesárea previa, con un feto único en presentación cefálica, de 37 semanas o más de embarazo)

\begin{tabular}{|c|c|}
\hline Total nacimientos & 106 \\
\hline Cesáreas & $49(53.8 \%)$ \\
\hline Subgrupo 5A: una única cesárea previa & $91(85.9 \%)$ \\
\hline Cesáreas subgrupo $5 \mathrm{~A}$ & $33(36.3 \%)$ \\
\hline $\begin{array}{l}\text { Partos vaginales tras una cesárea previa en } \\
\text { subgrupo } 5 \mathrm{~A}\end{array}$ & $58(63.7 \%)$ \\
\hline $\begin{array}{l}\text { Sin parto previo, periodo activo de parto, } \\
\text { finaliza en parto vaginal }\end{array}$ & 23 \\
\hline $\begin{array}{l}\text { Sin parto previo, periodo activo de parto, } \\
\text { finaliza en cesárea }\end{array}$ & 5 \\
\hline $\begin{array}{l}\text { Sin parto previo, inducción, finaliza en parto } \\
\text { vaginal }\end{array}$ & 21 \\
\hline Sin parto previo, inducción, finaliza en cesárea & 21 \\
\hline $\begin{array}{l}\text { Con parto previo, periodo activo de parto, } \\
\text { finaliza en parto vaginal }\end{array}$ & 3 \\
\hline $\begin{array}{l}\text { Con parto previo, periodo activo de parto, } \\
\text { finaliza en cesárea }\end{array}$ & 0 \\
\hline $\begin{array}{l}\text { Con parto previo, inducción, finaliza en parto } \\
\text { vaginal }\end{array}$ & 10 \\
\hline Con parto previo, inducción, finaliza en cesárea & 0 \\
\hline Cesárea previa a trabajo de parto & 7 \\
\hline Subgrupo 5B: dos o más cesáreas previas & $15(14.1 \%$ \\
\hline Cesáreas en subgrupo $5 \mathrm{~B}$ & $15(100 \%)$ \\
\hline $\begin{array}{l}\text { Partos vaginales tras una cesárea previa en } \\
\text { subgrupo } 5 B\end{array}$ & $0(0 \%)$ \\
\hline
\end{tabular}

(Tabla 5). Observamos que el $85.9 \%$ han tenido una única cesárea (subgrupo $5 \mathrm{~A}$ ), asociada o no a parto vaginal, mientras que el $14.1 \%$ presentan dos o más cesáreas (subgrupo 5B); en este último subgrupo, las pacientes no han tenido partos vaginales previos.

Realizar esta división en estos dos subgrupos es de gran utilidad para analizar la tasa de cesáreas de una manera más precisa. En el subgrupo $5 \mathrm{~A}$, la tasa de éxito de parto vaginal tras una cesárea es del $63.7 \%$, lo que mejora la tasa global que encontrábamos en el grupo 5, pero sigue estando en el límite bajo de lo descrito en la literatura, que sitúa la tasa de éxito de parto vaginal en las mujeres con una cesárea previa en torno al $60-80 \%{ }^{17}$. Dentro de este subgrupo $5 \mathrm{~A}$, aquellas pacientes que no presentan parto previo, podemos observar de nuevo la importancia de restringir al máximo el número de inducciones realizadas. Así, la tasa de éxito de parto vaginal en pacientes con una cesárea previa sin parto previo que inician trabajo de parto de manera espontánea es del $82.1 \%$, lo que se acerca a los mejores datos 
que podemos encontrar en otras series ${ }^{17}$ y a los que encontramos en nuestra serie en el grupo 1 (primíparas/multíparas con feto en cefálica, de 37 semanas o más de embarazo, que han iniciado el parto de manera espontánea). Sin embargo, en las pacientes con una cesárea previa sin parto previo a las que se practica una inducción, la tasa de éxito de parto vaginal en nuestro centro se reduce a un $50 \%$, valor muy alejado del observado en las mujeres que inician el trabajo de parto de manera espontánea. Un dato esperanzador que se desprende del análisis de las pacientes del subgrupo $5 \mathrm{~A}$ que solo presentan una cesárea es que aquellas que sí presentan parto vaginal previo, ya fuese antes o después de la cesárea, tienen una tasa de éxito de parto vaginal tras cesárea del $100 \%$ en nuestro centro, independientemente de cómo haya sido su inicio de parto. Estos datos mejoran los de Eden, et. al..$^{18}$, que publicaron una tasa de parto vaginal tras cesárea del $83 \%$ en aquellas pacientes que tenían un parto vaginal previo a la cesárea y de un $94 \%$ en las que habían experimentado ya un parto vaginal tras la cesárea.

Actualmente, de acuerdo con las guías de práctica clínica, haber tenido dos cesáreas previas no supone una contraindicación absoluta para intentar el parto vaginal, aunque sí puede suponer una contraindicación relativa ${ }^{19-22}$. Es importante señalar que, a pesar de que la tasa de éxito de parto vaginal descrita es similar a la de las mujeres que presentan una única cesárea previa $^{23-26}(65-85 \%)$, en un metaanálisis publicado en $2010^{27}$ se describió un aumento del riesgo de rotura uterina respecto a las que presentaban una única cesárea. Mientras las pacientes con una sola cesárea previa presentaban una tasa de rotura uterina en torno al $0.7 \%$, aquellas con dos cesáreas previas presentaban un $1.6 \%$. En nuestro centro, a las pacientes con dos o más cesáreas previas se les indica cesárea de manera electiva sin iniciar el trabajo de parto. Por ello, la tasa de cesáreas en este subgrupo 5B es del 100\%. Estas pacientes con dos o más cesáreas previas representan el $1.4 \%$ del total de los nacimientos de nuestro centro y el $5.3 \%$ del total de cesáreas que realizamos. Estos datos van en consonancia con el aumento de la tasa de cesáreas que se ha experimentado en los últimos años y subraya la importancia, de nuevo, de tratar de disminuir el número de cesáreas para reducir las pacientes que forman parte de este subgrupo. Además, modificar los protocolos y consensuar con la paciente la posibilidad de intentar realizar un parto vaginal a pesar de tener dos cesáreas previas, asumiendo los riesgos conocidos, puede ser una opción para reducir la tasa de cesáreas en este subgrupo.

Por último, para finalizar el análisis del grupo 5 , cabe señalar que en siete pacientes con una única cesárea previa se realizó cesárea antes del trabajo de parto. Seis de ellas revocaron la posibilidad de parto vaginal tras cesárea y solicitaron una cesárea electiva, suponiendo un $5.7 \%$ de las pacientes que integran el grupo 5 y un $2.1 \%$ del total de las cesáreas realizadas en nuestro centro en el año 2019. Como ya hemos comentado, las recomendaciones actuales no contraindican el parto vaginal tras una cesárea ${ }^{19-22}$. Instruir a las pacientes en la alta posibilidad de parto vaginal a pesar de haber tenido una cesárea previa debe ser también uno de los objetivos de nuestro servicio para reducir la tasa de cesáreas.

En cuanto a la tasa de cesáreas en los grupos 6 y 7 (gestantes nulíparas o multíparas, a término, con feto en podálica), es cercana al $100 \%$, cifras similares a las de otros estudios realizados en España ${ }^{11-13} y$ en otros países ${ }^{6,16,28}$ utilizando la clasificación Robson. Este dato refleja que en nuestro centro, actualmente, no se realizan partos de nalgas. La modificación de los protocolos como consecuencia de los resultados presentados en el Term Breech Tria ${ }^{29}$ y otros estudios ${ }^{30,31}$ ha llevado a una falta de entrenamiento del personal en los partos de nalgas y a la realización en el Hospital Doctor Peset de cesárea electiva en todas las gestaciones con feto en podálica. No obstante, en nuestro hospital se ofrece versión cefálica externa a aquellas pacientes con feto en presentación podálica y gestación a término, siempre que no esté contraindicada.

Los embarazos gemelares en nuestra muestra suponen un $2.2 \%$ del total de los nacimientos, con una tasa de cesáreas de $44.1 \%$. Se trata de un porcentaje de cesáreas inferior al presentado por otros estudios. Cabe recordar que el número de gestaciones múltiples va a aumentar en los próximos años como consecuencia de las técnicas de reproducción asistida, por lo que resulta importante mantener una buena tasa de cesáreas en este grupo para evitar un aumento en el número de cesáreas realizadas.

Por último, respecto al grupo 10 hay que destacar que nuestro centro no dispone de unidad de cuidados intensivos neonatales y solo atiende nacimientos pretérmino entre las semanas 32 y 37 . De esta manera, únicamente suponen el $2.9 \%$ de los nacimientos. Con ello, queremos indicar que en la tasa de cesáreas del Hospital Doctor Peset puede influir el hecho de no atender un grupo de riesgo como son los grandes prematuros. 


\section{Conclusiones}

El aumento de la tasa de cesáreas que se ha producido en las últimas décadas hace necesario la aplicación de sistemas que permitan auditar las cesáreas realizadas. El sistema de clasificación en 10 grupos de Robson es una herramienta útil y fácilmente implantable en los centros sanitarios para monitorizar e intervenir en los grupos de mujeres que más contribuyen al porcentaje de cesáreas. En el Hospital Doctor Peset, los que más aportan son el grupo $2 \mathrm{~A}$ y el grupo 5 , por lo que es fundamental una intervención urgente en ambos. El análisis de las indicaciones de inducción y la revisión de los protocolos ayudarán a reducir la tasa de cesáreas en nuestro centro, y nuevos estudios permitirán valorar la efectividad de las medidas implantadas.

\section{Financiamiento}

Los autores no han recibido financiamiento para la realización del trabajo.

\section{Conflicto de intereses}

Los autores declaran no tener ningún conflicto de intereses.

\section{Responsabilidades éticas}

Protección de personas y animales. Los autores declaran que para esta investigación no se han realizado experimentos en seres humanos ni en animales.

Confidencialidad de los datos. Los autores declaran que han seguido los protocolos de su centro de trabajo sobre la publicación de datos de pacientes.

Derecho a la privacidad y consentimiento informado. Los autores declaran que en este artículo no aparecen datos de pacientes.

\section{Bibliografía}

1. World Health Organization. Appropriate technology for birth. Lancet. 1985;2:436-7

2. Aelvoet W, Windey F, Molenberghs G, Verstraelen $H$, Van Reempts $P$, Foidart JM. Screening for inter-hospital differences in cesarean section rates in low-risk deliveries using administrative data: an initiative to improve the quality of care. BMC Health Serv Res. 2008;8:3.

3. Betrán AP, Merialdi M, Lauer JA, Bing-Shun W, Thomas J, Van Look P et al. Rates of caesarean section: analysis of global, regional and national estimates. Paediatr Perinat Epidemiol. 2007;21:98-113.

4. Anderson GM. Making sense of rising caesarean section rates. BMJ. 2004;329:696.

5. Portal Estadístico del Sistema Nacional de Salud. Disponible en: http:// www.msssi.gob.es/estadEstudios/estadísticas/sisInfSanSNS/home.ht
6. Robson MS. Classification of caesarean sections. Fetal Matern Med Rev. 2001;12:23-39.

7. Torloni MR, Betrán AP, Souza JP, Widmer M, Allen T, Gulmezoglu M, et al. Classifications for cesarean section: a systematic review. PLoS One. 2011;6:e14566.

8. Betran AP, Vindevoghel N, Souza JP, Guelmezoglu AM, Torloni MR. A systematic review of the Robson classification for caesarean section: what works, doesn't work and how to improve it. PLoS One. 2014;9:e97769.

9. World Health Organization. WHO statement on cesarean section rates. Geneva, Switzerland; 2015

10. FIGO Working Group on Challenges in Care of Mothers and Infants during Labour and Delivery. Best practice advice on the 10-group classification system for cesarean deliveries. Int J Gynaecol Obstet. 2016;135:232-3

11. Aguilar Redondo R, Manrique Fuentes G, Aisa Denaroso LM, Delgado Martínez L, González Acosta V, Aceituno Velasco L. Uso de la clasificación de Robson en un hospital comarcal de España para reducir la tasa de cesáreas. Rev Chil Obstet Ginecol. 2016;81:99-104.

12. Lorente CG, Raga FG. Implementation of the Robson 10-group classification system at Hospital de Manises: optimization of the cesarean delivery rate. Prog Obstet Ginecol. 2018;61:16-21.

13. Vengoechea PJ, Pérez A, Betrán A, Morey MDM, Borges MM, Alcácer P, et al. Clasificación de cesáreas por grupos de Robson en dos periodos comparativos en el Hospital de Manacor. Prog Obstet Ginecol. 2010;53:385-90.

14. Ray A, Jose S. Analysis of caesarean-section rates according to Robson's ten group classification system and evaluating the indications within the groups. Facilities. 2017;8:10.

15. Costa ML, Cecatti JG, Souza JP, Milanez HM, Gülmezoglu MA. Using a caesarean section classification system based on characteristics of the population as a way of monitoring obstetric practice. Reprod Health. 2010;7:13.

16. Kacerauskiene J, Bartuseviciene E, Railaite DR, Minkauskiene M, Bartusevicius A, Kliucinskas M, et al. Implementation of the Robson classification in clinical practice: Lithuania's experience. BMC Pregnancy Childbirth. 2017;17:432.

17. Sabol B, Denman MA, Guise J. Vaginal birth after cesarean: an effective method to reduce cesarean. Clin Obstet Gynecol. 2015;58:309-19.

18. Eden KB, McDonagh M, Denman MA, Marshall N, Emeis C, Fu R, et al. New insights on vaginal birth after cesarean: can it be predicted? Obstet Gynecol. 2010;116:967-81.

19. Royal College of Obstetricians and Gynaecologists. Birth after previous caesarean birth. Green-top Guideline 2015; No. 45.

20. Society of Obstetricians and Gynaecologists of Canada. SOGC clinical practice guidelines. Guidelines for vaginal birth after previous caesarean birth. Int J Gynaecol Obstet. 2005;89:319. Number 155 (Replaces guideline Number 147).

21. American College of Obstetricians and Gynecologists. ACOG Practice bulletin no. 205: Vaginal birth after previous cesarean delivery. Obstet Gynecol. 2019;133:e110-27.

22. Sociedad Española de Ginecología y Obstetricia (SEGO). Protocolos asistenciales en obstetricia. Parto vaginal tras cesárea. 2010.

23. Metz TD, Allshouse AA, Faucett AM, Grobman WA. Validation of vaginal birth after cesarean delivery prediction model in women with two prior cesareans. Obstet Gynecol. 2015;125:948.

24. Bretelle F, Cravello L, Shojai R, Roger V, D’Ercole C, Blanc B. Vaginal birth following two previous cesarean sections. Eur J Obstet Gynecol Reprod Biol. 2001;94:23-6.

25. Macones GA, Cahill A, Pare E, Stamilio DM, Ratcliffe S, Stevens E, et al. Obstetric outcomes in women with two prior cesarean deliveries: is vaginal birth after cesarean delivery a viable option? Am J Obstet Gynecol. 2005;192:1223-8.

26. Rotem R, Sela HY, Hirsch A, Samueloff A, Grisaru-Granovsky S, Rottenstreich $M$. The use of a strict protocol in the trial of labor following two previous cesarean deliveries: maternal and neonatal results. Eur J Obstet Gynecol Reprod Biol. 2020;252:387-92.

27. Tahseen S, Griffiths M. Vaginal birth after two caesarean sections (VBAC-2) - a systematic review with meta-analysis of success rate and adverse outcomes of VBAC-2 versus VBAC-1 and repeat (third) caesarean sections. BJOG. 2010;117:5-19.

28. Tanaka K, Mahomed K. The ten-group Robson classification: a single centre approach identifying strategies to optimise caesarean section rates. Obstet Gynecol Int. 2017;2017:5648938.

29. Hannah ME, Hannah WJ, Hewson SA, Hodnett ED, Saigal S, Willan AR, et al. Planned caesarean section versus planned vaginal birth for breech presentation at term: a randomised multicentre trial. Lancet. 2000;356:1375-83.

30. Hofmeyr GJ, Hannah ME. Planned caesarean section for term breech delivery. Cochrane Database Syst Rev. 2003;(3):CD000166.

31. Hehir MP. Trends in vaginal breech delivery. J Epidemiol Community Health. 2015;69:1237-9. 